\title{
Resultados da Intervenção Coronária Percutânea Primária em Pacientes do Sistema Único de Saúde e da Saúde Suplementar
}

\author{
Rodrigo Barretoํㅜㄹ \\ Silvio Gioppato1, João Batista de Freitas Guimarães', Evandro Karlo Pracchia Ribeiro1, \\ Júlio César Francisco Vardi' ${ }^{1}$ Patricia Teixeira da Silva1', Ricardo de Gasperi', \\ Leonardo Cao Cambra de Almeida' ${ }^{1}$, Leonardo dos Santos Coelho ${ }^{1}$, Roberto Simões de Almeida ${ }^{1}$
}

\section{RESUMO}

Introdução: A intervenção coronária percutânea (ICP) primária é a mais eficaz técnica de reperfusão no infarto agudo do miocárdio (IAM) e seu sucesso depende de múltiplos fatores. Este estudo teve como objetivo avaliar o perfil e comparar os resultados hospitalares da ICP primária entre os pacientes do Sistema Único de Saúde (SUS) e da saúde suplementar (SS). Métodos: Entre 2006 e 2010, 493 pacientes foram submetidos a ICP primária, sendo 220 tratados pelo SUS e 273 , pela SS. A técnica e a escolha do material ficaram a cargo dos operadores. Resultados: O grupo SUS tinha maior número de pacientes Killip > I. Doença coronária multiarterial, fluxo coronário pré-procedimento TIMI 0/1 e presença de colaterais para o vaso tratado não diferiram entre os grupos. O grupo SS utilizou mais cateteres de aspiração de trombos $(10 \%$ vs. $20,8 \% ; P<0,01)$ e inibidores da glicoproteína Ilb/Illa $(24,1 \%$ vs. $36,6 \%$; $P<0,01)$. Não houve diferença em relação ao tempo porta-balão (62,3 minutos vs. 64,2 minutos; $\mathrm{P}=0,91)$. Para os pacientes encaminhados de outros hospitais, no entanto, o tempo de transferência foi maior no SUS (400,8 minutos vs. 262,4 minutos; $\mathrm{P}<0,01)$. O sucesso da ICP e a ocorrência de eventos cardiovasculares e cerebrovasculares maiores (ECCAM) não diferiram entre os grupos $(6,3 \%$ vs. $6,2 \%$; $P=0,1)$. Idade, Killip III/IV e tempo de transferência foram as variáveis que melhor explicaram a ocorrência de ECCAM. Conclusões: As diferenças nos perfis clínico, angiográfico e do procedimento dos pacientes submetidos a ICP primária, atendidos pelo SUS e pela SS, não tiveram impacto nos ECCAM. O tempo de transferência, entretanto, elevado nos dois grupos e maior no grupo SUS, mostrou ser preditor independente de eventos adversos.

DESCRITORES: Angioplastia. Stents. Infarto do miocárdio. Sistema Único de Saúde. Saúde Suplementar.

\section{ABSTRACT}

Primary Percutaneous Coronary Intervention Outcomes in Patients from the Public versus the Private Healthcare Systems

Background: Primary percutaneous coronary intervention $(\mathrm{PCI})$ is the most effective reperfusion technique in acute myocardial infarction (AMI) and its success rate depends on many factors. This study aimed to assess the profile and to compare in-hospital outcomes of primary $\mathrm{PCl}$ among patients from the Public Healthcare System (PuHS) versus those from the Private Healthcare System (PrHS). Methods: From 2006 to 2010, 493 patients were submitted to primary $\mathrm{PCl}$, of which 220 were treated by the PuHS and 273 by the PrHS. Procedure technique and materials were left to the operator's discretion. Results: The PuHS group had a larger number of Killip $>1$ patients. Multivessel coronary disease, pre-procedure coronary flow TIMI $0 / 1$ and the presence of collaterals for the treated vessel did not differ between groups. The PrHS group used more thromboaspiration catheters $(10 \%$ vs. $20.8 \%$; $\mathrm{P}<0.01)$ and glycoprotein Ilb/IIla inhibitors $(24.1 \%$ vs. $36.6 \%$; $\mathrm{P}<0.01)$. There was no difference for door-to-balloon times (62.3 minutes vs. 64.2 minutes; $\mathrm{P}=0.91)$. For patients referred from other hospitals, however, transportation times were higher for PuHS patients (400.8 minutes vs. 262.4 minutes; $\mathrm{P}<0.01$ ). $\mathrm{PCl}$ success rates and the occurrence of major adverse cardiovascular and cerebrovascular events (MACCE) did not differ between groups $(6.3 \%$ vs. $6.2 \%$; $=0.1)$. Age, Killip III/IV and transportation time were the variables that best explained the occurrence of MACCE. Conclusions: Differences in the clinical, angiographic and procedure profile of patients undergoing primary $\mathrm{PCl}$ treated by the $\mathrm{PuHS}$ and the $\mathrm{PrHS}$ did not have an impact on MACCE. Transportation times, however, high in both groups and higher in the PuHS group, proved to be an independent predictor of adverse events.

KEY-WORDS: Angioplasty. Stents. Myocardial infarction. Unified Health System. Supplemental Health.
1 Hospital Bandeirantes - São Paulo, SP, Brasil.

Correspondência: Rodrigo Barreto. Rua Juquis, 301/113 - Bloco A Moema - São Paulo, SP, Brasil - CEP 04081-010

E-mail: barreto0202@hotmail.com

Recebido em: 3/7/2011 • Aceito em: 1ø/9/2011 
A s doenças cardiovasculares constituem o principal problema de saúde pública em países desenvolvidos, sendo responsáveis por até $45 \%$ das causas de morte nos países ocidentais. A doença isquêmica do coração representa $30 \%$ desse total, na maioria das vezes apresentando-se como síndrome coronária aguda. O infarto agudo do miocárdio com supradesnivelamento do segmento ST (IAMCSST) permanece como causa líder de mortalidade no Brasil, estimada em mais de 300 mil fatalidades anuais. ${ }^{1}$ As recomendações atuais indicam a realização de intervenção coronária percutânea (ICP) primária como tratamento de escolha do IAMCSST, desde que possa ser realizada em até 90 minutos da chegada do paciente ao hospital ou em até duas horas, quando há a necessidade de transferência para o procedimento..$^{2-4} \mathrm{O}$ benefício da ICP primária está relacionado a vários fatores, como maior taxa de patência do vaso-alvo (cerca de 90\%), possibilidade de simultaneamente tratar a estenose subjacente e menor taxa de complicações hemorrágicas associadas, quando comparada ao tratamento com fibrinolíticos..$^{5,6}$

Segundo os dados da Central Nacional de Intervenções Cardiovasculares (CENIC) da Sociedade Brasileira de Hemodinâmica e Cardiologia Intervencionista, foram realizadas 11.500 ICPs primárias no Brasil entre janeiro de 2006 e dezembro de 2010.7 A cada ano evidencia-se aumento progressivo do número dessa modalidade de ICP em relação às intervenções eletivas, de resgate ou facilitadas. ${ }^{7}$ Esse método terapêutico tem evoluído constantemente, alcançando taxas crescentes de sucesso relacionadas ao aperfeiçoamento dos stents coronários, dos novos dispositivos, como os cateteres de aspiração de trombos, e da farmacologia adjunta, com os novos tienopiridínicos e inibidores da glicoproteína IIb/IIla.

Os principais sistemas de saúde no Brasil, o Sistema Único de Saúde (SUS), público e disponível a toda a população, e a saúde suplementar (SS), formada por operadoras e seguradoras e restrita aos contribuintesbeneficiários, contemplam a ICP primária em seu rol de custeio. O SUS, no entanto, apresenta algumas limitações, como menor número de hospitais capacitados para realizar essa intervenção. O objetivo deste estudo foi saber se há diferenças nos perfis clínico e angiográfico e nos resultados hospitalares entre os pacientes desses dois sistemas de saúde submetidos a ICP primária e atendidos em um único centro de referência.

\section{MÉTODOS}

\section{Pacientes}

No período de agosto de 2006 a novembro de 2010, 493 pacientes foram submetidos consecutivamente a ICP primária no Serviço de Hemodinâmica e Cardiologia Intervencionista do Hospital Bandeirantes (São Paulo, SP), 220 tratados pelo SUS e 273 tratados pela SS. Foram comparadas as características clínicas, angiográficas e relacionadas ao procedimento, as taxas de sucesso e a incidência de eventos adversos até a alta hospitalar. Os dados foram coletados prospectivamente e armazenados em um banco de dados informatizado.

\section{Intervenção coronária percutânea}

As intervenções foram realizadas em sua quase totalidade por via femoral, sendo utilizadas as vias radial ou braquial como opção em poucos casos. A técnica e a escolha do material durante o procedimento ficaram a cargo dos operadores, assim como o uso de inibidores da glicoproteína Ilb/IIla e cateteres de tromboaspiração. Foi utilizada heparina não-fracionada no início do procedimento na dose de $70 \mathrm{U} / \mathrm{kg}$ a $100 \mathrm{U} / \mathrm{kg}$, exceto nos pacientes que já estavam em uso de heparina de baixo peso molecular. Nesses casos, adicionou-se a dose de $0,3 \mathrm{mg} / \mathrm{kg}$ da heparina de baixo peso molecular por via endovenosa, ajustada conforme o intervalo da última dose. Todos os pacientes receberam terapia antiplaquetária combinada, com ácido acetilsalicílico (dose de 100-200 mg/dia) e clopidogrel (dose de ataque de 300-600 mg e manutenção de $75 \mathrm{mg} / \mathrm{dia})$.

\section{Análise angiográfica e definições}

As análises foram realizadas em pelo menos duas projeções ortogonais por operadores experientes, com uso de angiografia quantitativa digital. Neste estudo foram utilizados os mesmos critérios angiográficos da CENIC. Para o tipo de lesão foram utilizados os critérios do American College of Cardiology/American Heart Association. ${ }^{8}$ Foram consideradas lesões longas aquelas com > $20 \mathrm{~mm}$ de comprimento. Para a classificação do fluxo coronário pré e pós-procedimento foi utilizada a classificação de TIMI. ${ }^{9}$ Sucesso do procedimento foi definido como obtenção de sucesso angiográfico (estenose residual < 30\%, com fluxo TIMI 3) e ausência de eventos cardiovasculares e cerebrovasculares adversos maiores (ECCAM), compreendendo morte, reinfarto, acidente vascular cerebral (AVC) e cirurgia de revascularização miocárdica (RM) de emergência. O reinfarto foi definido pela presença de alterações eletrocardiográficas (novo supradesnivelamento do segmento ST ou novas ondas Q) e/ou evidência angiográfica de oclusão do vaso-alvo. Foi considerada RM de emergência aquela realizada imediatamente após a ICP. ${ }^{2}$ Foram consideradas para análise a mortalidade geral (óbito por qualquer causa) e a mortalidade cardíaca (choque cardiogênico, insuficiência cardíaca, infarto agudo do miocárdio, ruptura cardíaca, arritmia e morte súbita) no período hospitalar.

O tempo porta-balão foi considerado como o intervalo medido entre a entrada do paciente no Serviço de Emergência do Hospital Bandeirantes até o início da reperfusão mecânica da artéria relacionada ao IAMCSST, quer seja por cateter-balão ou de tromboaspiração. O tempo de transferência foi considerado como o intervalo entre a chegada do paciente ao primeiro atendimento médico no hospital de origem e o início da 
reperfusão mecânica da artéria relacionada ao IAM no Serviço de Hemodinâmica e Cardiologia Intervencionista do Hospital Bandeirantes.

\section{Análise estatística}

Os dados armazenados em banco de dados com base Oracle foram plotados em planilhas Excel e analisados em programa estatístico SPSS, versão 15.0. As variáveis contínuas foram expressas em média \pm desvio padrão e as variáveis categóricas, em porcentuais. As associações entre as variáveis contínuas foram avaliadas pelos testes $t$ de Student, de Mann-Whitney ou ANOVA. As associações entre as variáveis categóricas foram avaliadas pelos testes qui-quadrado ou exato de Fischer nas tabelas de dimensão $2 \times 2$. Nas tabelas de maior dimensão, foram aplicados os testes qui-quadrado ou da razão de verossimilhança $(\mathrm{G})$. Foi adotado o nível de significância de pacientes $<0,05$. Modelos de regressão logística simples e múltipla foram aplicados para identificar preditores de ECCAM.

\section{RESULTADOS}

As características demográficas e clínicas dos dois grupos estão expostas na Tabela 1. O grupo SUS tinha um perfil de pacientes com menor grau de escolaridade, maior número de tabagistas, menor porcentual de revascularizações prévias, percutâneas ou cirúrgicas, e menor uso de estatinas e aspirina no período que antecedeu o evento isquêmico agudo. Apresentaram-se mais frequentemente com IAM em paredes anterior e inferior, e com maior número de pacientes em classe funcional Killip > I. Não ocorreram diferenças entre os dois grupos quanto a idade, sexo e porcentual de diabéticos.

Quanto às características angiográficas, expostas na Tabela 2, os pacientes não apresentaram diferenças em relação à maioria das características comparadas. O grupo SUS, entretanto, mostrou lesões um pouco menos complexas, de menor extensão e com menor acometimento de bifurcações. A presença de doença coronária multiarterial, o fluxo coronário pré-procedimento TIMI 0/1 e a presença de colaterais para o vaso tratado foram semelhantes entre os grupos.

Não houve diferenças significativas entre os grupos quanto a taxa de uso, diâmetro e comprimento dos stents, quantificação angiográfica das lesões-alvo pré e pós-procedimento, e número de vasos tratados por paciente. O grupo SS apresentou maior uso de cateter de aspiração de trombos $(10 \%$ vs. $20,8 \%$; $P<0,01)$ e de inibidores da glicoproteína IIb/IIla durante o procedimento $(24,1 \%$ vs. $36,6 \% ; P<0,01)$. O uso de stents farmacológicos foi exclusivo do grupo SS (Tabela 3).

Quanto aos intervalos avaliados, não houve diferenças em relação ao tempo porta-balão (62,3 minutos vs. $64,2$ minutos; $P=0,91)$. Para os pacientes transferidos, no entanto, o tempo de transferência foi maior no SUS $(400,8$ minutos vs. 262,4 minutos; $\mathrm{P}<0,01)$. O sucesso do procedimento foi semelhante entre os dois grupos $(91,4 \%$ vs. $93 \%$; $P=0,49)$ (Tabela 3 ).

Os desfechos hospitalares (Tabela 4) da ICP primária não apresentaram diferenças entre os grupos quanto a incidência de ECCAM $(6,3 \%$ vs. $6,2 \% ; P=0,1)$, ocorrência de $\mathrm{AVC}(0,5 \%$ vs. $0,4 \% ; \mathrm{P}=0,88)$, reinfarto $(1,4 \%$ vs. $2,6 \% ; P=0,35)$ e mortalidade hospitalar $(4,5 \%$ vs. $3,7 \% ; P=0,62)$. Todas as mortes foram de causa cardíaca e não foram realizadas cirurgias de emergência em nenhum dos grupos.

$\mathrm{Na}$ análise univariada, identificaram-se como preditores ECCAM a maior idade no procedimento, o sexo feminino, a classe funcional Killip III/IV e o tempo de transferência. Após análise multivariada, a maior idade no procedimento [odds ratio (OR) 1,05, intervalo de confiança de 95\% (IC 95\%) 1,02-1,1; P = 0,006], a classe funcional Killip III/IV (OR 3,83, IC 95\% 1,24$11,85 ; \mathrm{P}=0,019)$ e o tempo de transferência (OR 1, IC 95\% 1,0003-1,0015; $\mathrm{P}=0$ 0,003) foram as variáveis que melhor explicaram a presença de ECCAM hospitalares (Tabela 5).

\section{DISCUSSÃO}

O infarto agudo do miocárdio persiste como importante causa de morbidade e mortalidade, apesar dos avanços tanto no tratamento como na prevenção dessa condição, acarretando grandes custos aos sistemas de saúde. ${ }^{10}$ A ICP primária apresenta altas taxas de sucesso desde que realizada por operadores experientes, em centros de referência especializados, quando o diagnóstico e a transferência dos pacientes são realizados precocemente. $^{1-4}$

Nesta casuística de pacientes submetidos a ICP primária, a média de idade, que não diferiu entre os grupos, foi semelhante à encontrada no registro $\mathrm{CENIC}^{7} \mathrm{em}$ período de observação semelhante. A idade mais elevada, neste estudo, foi um preditor independente de ECCAM, com chance de eventos 5\% maior para cada ano de vida. A população idosa sabidamente tem apresentado menores taxas de sucesso e maiores riscos relacionados ao procedimento, que podem ser em parte explicados pela maior complexidade tanto clínica como angiográfica desses pacientes, como a coexistência de doenças sistêmicas graves, maior número de revascularizações cirúrgicas e infartos prévios, maior prevalência de doença multiarterial e de lesões coronárias complexas, e menor fração de ejeção do ventrículo esquerdo. ${ }^{11}$

A predominância do sexo masculino é compatível à encontrada em grandes registros, segundo os quais apenas 33\% das angioplastias primárias são realizadas em mulheres. ${ }^{12,13}$ Observou-se maior prevalência de dislipidemia, de revascularizações prévias e de uso de estatinas e ácido acetilsalicílico no grupo SS e maior prevalência de tabagismo no grupo SUS. É possível que esses resultados também estejam relacionados às di- 
TABELA 1

Características demográficas e clínicas

\begin{tabular}{|c|c|c|c|}
\hline Característica & $\begin{array}{c}\text { SUS } \\
(n=220)\end{array}$ & $\begin{array}{c}\text { SS } \\
(n=273)\end{array}$ & Valor de $\mathbf{P}$ \\
\hline Idade em anos, média \pm desvio padrão & $60,8 \pm 11,5$ & $61,8 \pm 13,8$ & 0,4 \\
\hline Sexo masculino, n (\%) & $160(72,7)$ & $195(71,4)$ & 0,75 \\
\hline Escolaridade, n (\%) & & & $<0,01$ \\
\hline Não-alfabetizado & $3(1,4)$ & 0 & \\
\hline 1a à $4^{a}$ séries & $48(21,8)$ & $32(11,7)$ & \\
\hline 5a à $8^{a}$ séries & $70(31,8)$ & $40(14,7)$ & \\
\hline 2o grau & $70(31,8)$ & $84(30,8)$ & \\
\hline Superior & $2(0,9)$ & $72(26,4)$ & \\
\hline Mestrado ou doutorado & 0 & $2(0,7)$ & \\
\hline Não sabe/sem declaração & $27(12,3)$ & $43(15,8)$ & \\
\hline Hipertensão arterial, n (\%) & $156(70,9)$ & $176(64,5)$ & 0,13 \\
\hline Dislipidemia, n (\%) & $32(14,5)$ & $86(31,5)$ & $<0,01$ \\
\hline Diabetes melito, $\mathrm{n}(\%)$ & $61(27,7)$ & $64(23,4)$ & 0,28 \\
\hline Tabagismo, n (\%) & $70(31,8)$ & $66(24,2)$ & 0,06 \\
\hline IAM prévio, n (\%) & $20(9,1)$ & $27(9,9)$ & 0,76 \\
\hline ICP prévia, n (\%) & $14(6,4)$ & $31(11,4)$ & 0,055 \\
\hline RM prévia, n (\%) & $4(1,8)$ & $17(6,2)$ & 0,02 \\
\hline \multicolumn{4}{|l|}{ Tratamento farmacológico, n (\%) } \\
\hline Estatina & $20(9,1)$ & $57(20,8)$ & $<0,01$ \\
\hline AAS & $36(16,4)$ & $64(23,4)$ & 0,054 \\
\hline Clopidogrel & $8(3,6)$ & $8(2,9)$ & 0,77 \\
\hline Killip, n (\%) & & & $<0,01$ \\
\hline I & $147(70,3)$ & $215(81,1)$ & \\
\hline$\|$ & $43(20,6)$ & $23(8,7)$ & \\
\hline III & $4(1,9)$ & $5(1,9)$ & \\
\hline IV & $15(7,2)$ & $22(8,3)$ & \\
\hline Localização do IAM, n (\%) & & & 0,01 \\
\hline Anterior & $87(41,6)$ & $97(36,6)$ & \\
\hline Inferior & $81(38,8)$ & $84(31,7)$ & \\
\hline Ínfero-látero-dorsal & $7(3,3)$ & $32(12,1)$ & \\
\hline Lateral & $5(2,4)$ & $6(2,3)$ & \\
\hline Dorsal & $2(1)$ & $2(0,8)$ & \\
\hline Ínferodorsal & $17(8,1)$ & $17(6,4)$ & \\
\hline Ânterolateral & $10(4,8)$ & $27(10,2)$ & \\
\hline
\end{tabular}

AAS = ácido acetilsalicílico; IAM = infarto agudo do miocárdio; ICP = intervenção coronária percutânea; $\mathrm{n}$ = número de pacientes; RM = cirurgia de revascularização miocárdica; SS = saúde suplementar; SUS = Sistema Único de Saúde.

ferenças socioeconômicas e de acesso à saúde, tanto no aspecto preventivo como no adequado acompanhamento das doenças cardiovasculares.

A classificação funcional Killip-Kimball foi diferente entre os grupos, com maior prevalência de classes $>$ I no grupo SUS. À semelhança de outras casuísticas, como a análise conjunta dos estudos PAMI ${ }^{14}$, as classes III/IV constituíram-se em preditores independentes de ECCAM, apresentando chance quatro vezes maior de ocorrência de desfechos adversos que os pacientes em Killip I/II.
Apesar de o implante de stents farmacológicos no cenário de ICP primária estar respaldado em várias publicações ${ }^{2,4,15,16}$, seu uso em nosso meio continua baixo $(<10 \%)$, segundo os dados do registro CENIC. ${ }^{7}$ Esse fato tem respaldo em aspectos de financiamento dos sistemas de saúde, em que o SUS ainda não contempla essa tecnologia em seu rol de custeio e a saúde suplementar impõe restrições a sua utilização. Além dessas razões, a prioridade do restabelecimento do fluxo anterógrado coronário no vaso ocluído e a perda de tempo adicio- 
TABELA 2

Características angiográficas

\begin{tabular}{|c|c|c|c|}
\hline Característica & $\begin{array}{c}\text { SUS } \\
\text { (220 pacientes/261 lesões) }\end{array}$ & $\begin{array}{c}\text { SS } \\
\text { (273 pacientes/333 lesões) }\end{array}$ & Valor de $\mathbf{P}$ \\
\hline Vasos acometidos, n (\%) & & & 0,75 \\
\hline Um & $102(47)$ & $133(49,4)$ & \\
\hline Dois & $71(32,7)$ & $84(31,2)$ & \\
\hline Três & $35(16,1)$ & $44(16,4)$ & \\
\hline Lesões calcificadas, n (\%) & $120(46)$ & $166(49,7)$ & 0,35 \\
\hline Lesões tipo B2/C, n (\%) & $192(73,6)$ & $270(81,1)$ & 0,18 \\
\hline Lesões longas, n (\%) & $23(8,8)$ & $45(13,5)$ & 0,08 \\
\hline Bifurcações, n (\%) & $9(3,4)$ & $28(8,4)$ & 0,01 \\
\hline Fluxo TIMI pré-ICP, n (\%) & & & 0,54 \\
\hline TIMI 0/1 & $180(69)$ & $229(68,6)$ & \\
\hline TIMI 2/3 & $81(31)$ & $105(31,5)$ & \\
\hline Circulação colateral, n (\%) & $38(43,2)$ & $28(35,5)$ & 0,25 \\
\hline Vaso tratado, n (\%) & & & 0,77 \\
\hline $\mathrm{CD}$ & $100(38,2)$ & $120(35,9)$ & \\
\hline $\mathrm{DA}$ & $106(40,6)$ & $139(41,6)$ & \\
\hline $\mathrm{CX}$ & $30(12,6)$ & $46(14,7)$ & \\
\hline TCE & $3(1,1)$ & $0(0)$ & \\
\hline
\end{tabular}

$\mathrm{CD}=$ artéria coronária direita; $\mathrm{CX}=$ artéria coronária circunflexa; $\mathrm{DA}=$ artéria descendente anterior; $\mathrm{ICP}=$ intervenção coronária percutânea; TCE = tronco de coronária esquerda; TIMI = Thrombolysis in Myocardial Infarction; SS = saúde suplementar; SUS = Sistema Único de Saúde.

nal para efetuar análise clínica minuciosa para identificar contraindicações relativas ao uso de antiagregação dupla ou submeter o enfermo a entraves burocráticos são absolutamente não recomendadas. ${ }^{2}$ Dessa forma, mesmo no grupo SS, em que há a possibilidade do uso de stents farmacológicos, sua utilização foi baixa (8,5\%).

A aspiração de trombos durante ICP primária apresenta recomendação nível de evidência IIA, com nível de evidência B nas principais diretrizes atuais ${ }^{2-4}$, resultando em melhores taxas de reperfusão e de desfechos clínicos em relação à ICP sem aspiração de trombos. Neste estudo, observou-se que o uso desse dispositivo foi baixo ( $10 \%$ no SUS e $20,8 \%$ no SS), e ficou a critério do operador. Considerando os benefícios clínicos e angiográficos demonstrados por esses dispositivos em publicações recentes, acredita-se que o uso rotineiro desse dispositivo deva ser encorajado. ${ }^{15-19}$

O uso de inibidores da glicoproteína IIb/IIla e de cateteres de aspiração também foi maior no grupo SS. A decisão de utilização desses recursos também ficou a critério dos operadores, que levam em conta a carga de trombo e as alterações de fluxo no vaso como critérios de indicação. Acredita-se que as taxas do uso de inibidores da glicoproteína Ilb/IIla neste trabalho condizem com suas recomendações atuais, segundo as quais seu uso deve ser restrito a situações específicas. ${ }^{4}$

O tempo porta-balão e o tempo de transferência estão diretamente relacionados às taxas de mortalida- de no atendimento ao paciente com IAM, devendo o primeiro ser $<90$ minutos e o segundo, $<2$ horas, para que os benefícios dessa terapia de reperfusão sejam plenamente atingidos. ${ }^{2-4} \mathrm{O}$ tempo porta-balão em nossa instituição está adequado ao preconizado pelas diretrizes, independentemente da fonte pagadora (SUS ou SS), sendo derivado de protocolos gerenciados de dor torácica e atendimento ao paciente com IAM por equipes multidisciplinares do pronto-socorro e do Serviço de Terapia Intensiva e Hemodinâmica, e que disponibiliza in loco equipe de enfermagem e cardiologista intervencionista 24 horas por dia, 7 dias por semana.

Quanto aos pacientes provenientes de outros hospitais, o tempo de transferência foi elevado em ambos os grupos e maior no SUS. Esse fato pode ter ocorrido por demora no atendimento primário, indisponibilidade ou dificuldade de obtenção imediata de meio de transporte adequado (ambulância-UTI), pelo trânsito difícil de uma grande metrópole, como São Paulo, e pelas grandes distâncias entre os centros de atendimento primário e o centro de referência. Há ainda as diferenças de definição, pois considerou-se o tempo de transferência desde a chegada do paciente ao primeiro hospital de atendimento até o início da reperfusão mecânica, ao passo que na literatura o tempo de transferência refere-se ao período entre o diagnóstico no hospital inicial e a chegada do paciente ao laboratório de hemodinâmica no hospital de referência. O tempo 
TABELA 3

Características dos procedimentos

\begin{tabular}{|c|c|c|c|}
\hline Característica & $\begin{array}{c}\text { SUS } \\
\text { (220 pacientes/261 lesões) }\end{array}$ & $\begin{array}{c}\text { SS } \\
\text { (273 pacientes/333 lesões) }\end{array}$ & Valor de $\mathbf{P}$ \\
\hline Vasos tratados/paciente, média \pm desvio padrão & $1,2 \pm 0,5$ & $1,2 \pm 0,5$ & 0,37 \\
\hline Uso de stent, n (\%) & $228(87,3)$ & $293(87,9)$ & 0,99 \\
\hline Stents convencionais & $228(100)$ & $268(91,5)$ & 0,88 \\
\hline Stents farmacológicos & 0 & $25(8,5)$ & 0,01 \\
\hline Diâmetro dos stents, mm & $3,11 \pm 0,49$ & $3,09 \pm 0,52$ & 0,77 \\
\hline Comprimento dos stents, $\mathrm{mm}$ & $21,1 \pm 7$ & $19,9 \pm 6,5$ & 0,58 \\
\hline Inibidores da glicoproteína IIb/IIla, n (\%) & $53(24,1)$ & $100(36,6)$ & $<0,01$ \\
\hline Tromboaspiração, n (\%) & $22(10)$ & $57(20,8)$ & $<0,01$ \\
\hline Fluxo TIMI pós-ICP, n (\%) & & & 0,2 \\
\hline TIMI 0/1 & $7(3,1)$ & $3(1)$ & \\
\hline TIMI 2/3 & $221(96,9)$ & $290(98,9)$ & \\
\hline Diâmetro da estenose pré, \% & $96,1 \pm 9,7$ & $94,8 \pm 10,8$ & 0,5 \\
\hline Diâmetro da estenose pós, \% & $1 \pm 7,4$ & $1,5 \pm 6,8$ & 0,69 \\
\hline Tempo porta-balão, minutos & $62,3 \pm 192,9$ & $64,2 \pm 169,2$ & 0,91 \\
\hline Tempo de transferência, minutos & $400,8 \pm 403,3$ & $262,4 \pm 364,8$ & $<0,01$ \\
\hline Sucesso do procedimento, n (\%) & $201(91,4)$ & $254(93)$ & 0,49 \\
\hline
\end{tabular}

TABELA 4

Desfechos clínicos na fase hospitalar

\begin{tabular}{lccc}
\hline Característica & $\begin{array}{c}\text { SUS } \\
(\mathbf{n}=\mathbf{2 2 0})\end{array}$ & $\begin{array}{c}\text { SS } \\
(\mathbf{n}=\mathbf{2 7 3})\end{array}$ & Valor de P \\
\hline ECCAM, n (\%) & $14(6,3)$ & $17(6,2)$ & 0,1 \\
Óbito hospitalar, n (\%) & $10(4,5)$ & $10(3,7)$ & 0,62 \\
AVC, n (\%) & $1(0,5)$ & $1(0,4)$ & 0,88 \\
Reinfarto, n (\%) & $3(1,4)$ & $7(2,6)$ & 0,35 \\
RM de emergência, n (\%) & 0 & 0 & NA \\
\cline { 2 - 3 } AVC = acidente vascular cerebral; ECCAM = eventos cardíacos e cerebrovasculares adversos maiores; $\mathrm{n}=$ número de pacientes; NA = \\
não-aplicável; RM = cirurgia de revascularização miocárdica; SS = saúde suplementar; SUS = Sistema Único de Saúde. \\
\hline
\end{tabular}

TABELA 5

Preditores independentes de eventos cardíacos e cerebrovasculares adversos maiores

\begin{tabular}{lcr}
\hline Fatores & OR & IC 95\% \\
\hline Idade & 1,05 & $1,02-1,1$ \\
Killip III/IV & 3,83 & $1,24-11,85$ \\
Tempo de transferência & 1 & $1,0003-1,0015$ \\
\hline
\end{tabular}

IC $95 \%$ = intervalo de confiança de $95 \%$; OR = odds ratio.

de transferência foi, nesta casuística, um preditor independente de ECCAM, e a chance de os eventos ocorrerem aumentou em $0,1 \%$ a cada minuto do tempo mensurado.
As taxas de sucesso do procedimento em ambos os grupos foram atingidas de acordo com as Diretrizes atuais $^{2-4}$, apesar de o grupo SS apresentar tempo de transferência inferior e médias superiores do uso de 
cateter de aspiração e de inibidores da glicoproteína IIb/IIla. A incidência de ECCAM, reinfarto, AVC e óbito foi baixa e semelhante entre os grupos.

\section{Limitações do estudo}

São limitações deste estudo a análise retrospectiva dos dados, sua realização em um único centro e a ausência de seguimento tardio.

\section{CONCLUSÕES}

Apesar das diferenças nos perfis clínico, angiográfico e do procedimento encontradas entre os pacientes submetidos a ICP primária atendidos pelo SUS e pela SS, os eventos clínicos hospitalares não diferiram entre os grupos. A idade, a classe funcional Killip III/IV e o tempo de transferência foram preditores independentes de ECCAM hospitalares nesses pacientes.

\section{CONFLITO DE INTERESSES}

Os autores declaram não haver conflito de interesses relacionado a este manuscrito.

\section{REFERÊNCIAS}

1. Ribeiro H, Ribeiro ER. Intervenção coronária percutânea em pacientes com síndrome coronariana aguda com supradesnivelamento do segmento ST. In: Ribeiro ER, Martinez EE. Hemodinâmica e cardiologia intervencionista. Barueri (SP): Manole; 2008.

2. Mattos LA, Lemos Neto PA, Rassi A Jr, Marin-Neto JA, Sousa AGMR, Devito FS, et al. Diretrizes da Sociedade Brasileira de Cardiologia - Intervenção Coronária Percutânea e Métodos Adjuntos Diagnósticos em Cardiologia Intervencionista (II Edição - 2008). Arq Bras Cardiol. 2008;91(6 Supl 1):1-58.

3. Piegas LS, Feitosa G, Mattos LA, Nicolau JC, Rossi Neto JM, Timerman A, et al. Sociedade Brasileira de Cardiologia. Diretriz da Sociedade Brasileira de Cardiologia sobre Tratamento do Infarto Agudo do Miocárdio com Supradesnível do Segmento ST. Arq Bras Cardiol. 2009;93(6 Supl 2):e179-e264.

4. European Association for Percutaneous Cardiovascular Interventions; Wijns W, Kolh P, Danchin N, Di Mario C, FalkV, Folliguet T, et al. The Task Force on Myocardial Revascularization of the European Society of Cardiology (ESC) and the European Association for Cardio-Thoracic Surgery (EACTS). Guidelines on myocardial revascularization. Eur Heart J. 2010; 31(20):2501-55

5. Keeley EC, Boura JA, Grines CL. Primary angioplasty versus intravenous thrombolytic therapy for acute myocardial infarction: a quantitative review of 23 randomized trials. Lancet. 2003; 361(9351):13-20.

6. Werf F, Bax J, Betriu A, Blomstrom-Lundqvist C, Crea F, Falk $V$, et al. Management of acute myocardial infarction in patients presenting with persistent ST-segment elevation: the Task Force on the Management of ST-Segment Elevation Acute Myocardial Infarction of the European Society of Cardiology. Eur Heart J. 2008;29(23):2909-45.

7. Matte BS, Bergoli LCC, Balvedi JA, Zago AC. Perfil da inter- venção coronária percutânea no infarto agudo do miocárdio com supradesnivelamento do segmento ST no Brasil de 2006 a 2010 - Registro CENIC. Rev Bras Cardiol Invasiva. 2011; 19(2):131-7.

8. Smith SC Jr, Feldman TE, Hirshfeld JW Jr, Jacobs AK, Kern MJ, King SB 3 $3^{\text {rd }}$, et al. ACC/AHA/SCAI 2005 guideline update for percutaneous coronary intervention: a report of the American College of Cardiology/American Heart Association Task Force on Practice Guidelines (ACC/AHA/SCAI writing Committee to Update 2001 Guideline for Percutaneous Coronary Intervention). Circulation. 2006;113(7):e166-286.

9. TIMI Study Group. The Thrombolysis in Myocardial Infarction (TIMI) trial. Phase I findings. N Engl J Med. 1985;312(14): 932-6.

10. Brasil. Ministério da Saúde. DATASUS. Informações de saúde [Internet]. [citado 2011 mar.14]. Disponível em: http://www2. datasus.gov.br/DATASUS/index.php?area $=02$

11. Thomas Junior NR, Cantarelli MJC, Castello Junior HJ, Gioppato $S$, Gonçalves R, Guimarães JBF, et al. Resultados hospitalares da intervenção coronária percutânea primária no infarto agudo do miocárdio em pacientes com mais de 80 anos. Rev Bras Cardiol Invasiva. 2010;18(1):17-23.

12. Glaser R, Herrmann HC, Murphy SA, Demopoulos LA, DiBattiste $\mathrm{PM}$, Cannon $\mathrm{CP}$, et al. Benefit of an early invasive management strategy in women with acute coronary syndromes. JAMA. 2002;288(24):3124-9.

13. Mueller C, Neumann FJ, Roskamm H, Buser P, Hodgson JM, Perruchoud AP, et al. Women do have an improved longterm outcome after non-ST-elevation acute coronary syndromes treated very early and predominantly with percutaneous coronary intervention: a prospective study in 1,450 consecutive patients. J Am Coll Cardiol. 2002;40(2):245-50.

14. DeGeare VS, Stone GW, Brodie BR, Cox DA, Garcia E. Angiographic and clinical characteristics associated with increased in-hospital mortality in elderly patients with acute myocardial infarction undergoing percutaneous intervention (a pooled analysis of the Primary Angioplasty in Myocardial Infarction trials). Am J Cardiol. 2000;86(1):30-4.

15. De Luca G, Stone GW, Suryapranata H, Laarman GJ, Menichelli M, Kaiser C, et al. Efficacy and safety of drug eluting stents in ST-segment elevation myocardial infarction: a meta-analysis of randomized trials. Int J Cardiol. 2009;133(2):213-22.

16. Kastrati A, Dibra A, Spaulding C, Laarman GJ, Menichelli M, Valgimigli $M$, et al. Meta-analysis of randomized trials on drug-eluting stents vs. baremetal stents in patients with acute myocardial infarction. Eur Heart J. 2007;28(22):2706-13.

17. Svilaas T, Vlaar PJ, van der Horst IC, Diercks GFH, de Smet BJGL, van den Heuvel AF, et al. Thrombus aspiration during primary percutaneous coronary intervention. $\mathrm{N}$ Engl J Med. 2008;358(6):557-67.

18. Vlaar PJ, Svilaas T, van der Horst IC, Diercks GF, Fokkema $\mathrm{ML}$, de Smet BJ, et al. Cardiac death and reinfarction after 1 year in the Thrombus Aspiration during percutaneous coronary intervention in Acute Myocardial Infarction Study (TAPAS): a 1-year follow-up study. Lancet. 2008;371(9628): 1915-20.

19. Burzotta F, De Vita M, Gu YL, Isshiki T, Lefèvre T, Kaltoft $A$, et al. Clinical impact of thrombectomy in acute ST-elevation myocardial infarction: an individual patient-data pooled analysis of 11 trials. Eur Heart J. 2009;30(18):2193-203. 